Published in final edited form as:

Cancer Causes Control. 2015 August ; 26(8): 1055-1063. doi:10.1007/s10552-015-0598-0.

\title{
Menopausal hormone therapy and mortality among endometrial cancer patients in the NIH-AARP Diet and Health Study
}

\author{
Ashley S. Felix ${ }^{1,2}$, Hannah Arem ${ }^{3}$, Britton Trabert ${ }^{1}$, Gretchen L. Gierach ${ }^{1}$, Yikyung Park ${ }^{3,4}$, \\ Ruth M. Pfeiffer ${ }^{5}$, and Louise A. Brinton ${ }^{1}$ \\ ${ }^{1}$ Hormonal and Reproductive Epidemiology Branch, Division of Cancer Epidemiology and \\ Genetics, National Cancer Institute, National Cancer Institute, 9609 Medical Center Drive, \\ Bethesda, MD 20892-9774 \\ ${ }^{2}$ Cancer Prevention Fellowship Program, Division of Cancer Prevention, National Cancer Institute, \\ 9609 Medical Center Drive, Bethesda, MD 20892-9774 \\ ${ }^{3}$ Nutritional Epidemiology Branch, Division of Cancer Epidemiology and Genetics, National \\ Cancer Institute, 9609 Medical Center Drive, Bethesda, MD 20892-9774 \\ ${ }^{5}$ Biostatistics Branch, Division of Cancer Epidemiology and Genetics, National Cancer Institute, \\ 9609 Medical Center Drive, Bethesda, MD 20892-9774
}

\section{Abstract}

Background-While menopausal hormone therapy (MHT) is an established endometrial cancer risk factor, its relationship with mortality among endometrial cancer patients is understudied.

\begin{abstract}
Methods-Within the NIH-AARP Diet and Health Study, we examined associations of prediagnosis MHT use with 10-year all-cause and endometrial cancer-specific mortality among 890 endometrial cancer patients. We used Cox proportional hazards regression models to estimate hazard ratios (HRs) and 95\% confidence intervals (CIs) adjusted for tumor characteristics, treatment, and other risk factors.
\end{abstract}

Results-Endometrial cancer cases were diagnosed a median of 4.6 years (range $0.0-10.1$ years) after the second risk factor questionnaire was completed. We identified a total of 241 deaths, of which, 104 were due to endometrial cancer. Compared with no MHT use, pre-diagnosis use of estrogen plus progestin therapy (EPT)-only was associated with lower 10-year all-cause ( $\mathrm{HR}=0.65,95 \% \mathrm{CI}=0.43-0.99$, based on 29 deaths) and endometrial cancer-specific mortality ( $\mathrm{HR}=0.51,95 \% \mathrm{CI}=0.26-0.98$, based on 11 deaths). Recency of MHT use, assessed approximately 5 years prior to the endometrial cancer diagnosis, was associated with mortality. Compared with non-MHT users, former ET users had higher all-cause (HR=1.71, 95\% CI=1.02-2.88, based on 18 deaths) and endometrial cancer-specific mortality $(\mathrm{HR}=2.17,95 \% \mathrm{CI}=0.96-4.90$, based on 8 deaths), whereas current EPT users had non-significant lower risks of death.

Corresponding author: Ashley S. Felix, Hormonal and Reproductive Epidemiology Branch, National Cancer Institute, 9609 Medical Center Drive, Room 7E214, Bethesda, MD 20892-9774, Phone: 1-240-276-7325, Fax: 1-240-276-7838, ashley.felix@ nih.gov.

${ }^{4}$ Current affiliation: Division of Public Health Sciences, Washington University School of Medicine, St. Louis, MO 63110

The authors declare that there are no conflicts of interest. 
Conclusion-Based on small numbers, we observed that pre-diagnosis use of EPT was related to lower mortality among endometrial cancer patients. Future studies examining the biological mechanisms underlying this association are warranted.

\section{Keywords}

estrogen plus progestin; endometrial cancer; survival

\section{INTRODUCTION}

Menopausal hormone therapy (MHT) is an established endometrial cancer risk factor [1]. Endometrial cancer risk is 2-10 times higher among estrogen therapy (ET) users [2] whereas risk among users of estrogen plus progestin therapy (EPT) depends on the duration that progestin is supplied [3]. Short duration formulations, also termed sequential or cyclic, add a progestin component for less than 15 days per cycle and increase endometrial cancer risk by approximately $15 \%$ compared with non-use [4]. Long duration formulations, also termed continuous, provide daily progestin and have been shown to lower endometrial cancer by approximately $20 \%$ [1].

Most of the literature related to pre-diagnosis MHT use and mortality among endometrial cancer cases has focused on ET with inconsistent findings: some report no significant relationship [5-7] while others report lower mortality among ET users [8-13]. Among the latter group of studies, only two adjusted for potentially favorable tumor characteristics associated with ET, including less invasive stage and lower grade $[9,11]$. The inconsistency in the previous literature is likely related to variable ascertainment of ET use (medical record information vs. self-report at time of diagnosis), differences in the inclusion of potential confounders, and small sample sizes which may have led to an inability to detect mortality differences between ET users and non-users.

Only one previous study has investigated associations between pre-diagnosis EPT and mortality among 683 endometrial cancer cases [13]. EPT (any duration of progestin) was not associated with survival among the case group relative to the expected survival estimated from the general population. Further, relative survival was unrelated to sequential EPT and no estimates for continuous EPT were provided, presumably due to low number of users in this category. To evaluate further the relationship of pre-diagnosis MHT use and mortality among endometrial cancer cases, we utilized data from the large, prospective National Institutes of Health (NIH)-AARP Diet and Health Study which assessed detailed information on MHT formulation, other endometrial cancer risk factors, and endometrial tumor characteristics.

\section{MATERIALS AND METHODS}

\section{Study population}

The NIH-AARP Diet and Health Study has been described previously [14]. Briefly, a baseline questionnaire was mailed in 1995-1996 to 3.5 million AARP members 50-71 years of age who resided in six states (California, Florida, Louisiana, New Jersey, North Carolina, 
and Pennsylvania) or two metropolitan areas (Atlanta, GA and Detroit, MI). Between 1996 and 1997, a second risk factor questionnaire, which assessed MHT use in greater detail, was sent to baseline questionnaire respondents who did not have self-reported colon, breast, or prostate cancer at baseline. Our cohort consisted of 1,049 endometrial cancer cases diagnosed a median of 4.6 years (range: $0.0-10.1$ years) after the second risk factor questionnaire was completed. Of these, we excluded women who reported a hysterectomy before baseline ( $\mathrm{n}=14)$, had unknown hysterectomy status $(\mathrm{n}=12)$, menstrual periods that stopped due to surgery $(n=7)$, premenopausal women $(n=63)$, women with unknown menopausal status $(n=5)$, women that developed non-epithelial cancer during follow-up $(n=29)$, and women with unknown type of MHT $(n=29)$ leaving 890 incident endometrial cancers with data from the second risk factor questionnaire available for analysis. The NIHAARP Diet and Health Study was approved by the Special Studies Institutional Review Board of the U.S. National Cancer Institute and all participants gave informed consent by virtue of completing and returning the questionnaire.

\section{Cancer incidence and treatment information}

Incident endometrial cancers were identified by probabilistic linkages with cancer registries in the original recruitment areas and two common states of relocation (Arizona and Texas). Completeness of case ascertainment in the NIH-AARP Diet and Health cohort is high Michaud and colleagues [15] reported sensitivity of $90 \%$ and specificity of $99.5 \%$ with respect to identification of cases by cancer registry linkage.

Date of cancer diagnosis, histology, stage, grade, and first course of treatment were available from cancer registries. Histology was defined using the International Classification of Diseases for Oncology (ICD-O 3rd Edition) and American Joint Committee on Cancer Staging System was used for classification of stage. Endometrial cancer cases with the following histology codes were included for analysis: endometrioid $(8380,8382,8383)$, adenocarcinoma (8140, 8210, 8560, 8570), mucinous adenocarcinoma $(8480,8482)$, clear cell (8310), serous $(8441,8460,8461)$, mixed cell $(8323)$, carcinosarcoma $(8950,8951$, 8980) and other epithelial histology types (8000, 8010, 8012, 8022, 8041, 8050, 8071, 8076, $8255,8260,8320)$. Tumor grade is only applicable for endometrioid and adenocarcinoma histologic subtypes [16]. Therefore, we created a summary variable incorporating both histology and tumor grade: low-grade (grades 1 and 2) endometrioid or adenocarcinoma, high-grade (grade 3) endometrioid or adenocarcinoma, serous, and other, which included clear cell, carcinosarcoma, and other low-frequency histology subtypes.

\section{Mortality ascertainment}

Addresses for cohort members were updated periodically in response to information provided by the participants and through the National Change of Address database. Vital status and causes of death were ascertained by linkage to the U.S. Social Security Administration Death Master File and the National Death Index Plus through December 31, 2011. ICD-9 and ICD-10 codes were used to identify deaths due to endometrial cancer (ICD-9: 179 and 182; ICD-10: C54-C55). Accuracy of vital status ascertainment in this cohort is greater than $95 \%$ [17]. 


\section{Menopausal hormone therapy and covariate assessment}

As previously described [18], detailed MHT information, including dates of first and last use, total duration of use, regimen, usual dose, and the name of the pill taken for the longest time was collected on the second risk factor questionnaire. Women were classified as using EPT-only if the reported dates of estrogen and progestin use overlapped or were within 90 days of each other. Sequential EPT was defined as progestin delivered for less than 15 days per cycle. Although continuous EPT is typically defined as progestin delivered for 25 or more days per cycle, we included women who used progestin for 15-25 days per cycle $(n=36)$ in our definition of continuous EPT. Inclusion of these women did not materially change our estimates.

Women who reported using ET and EPT without overlapping dates or with unknown duration of progestin were included in a separate category, and include: use of EPT after use of ET ( $n=86)$ or after use of progestin-only (PT, $n=4)$; use of EPT before use of ET ( $n=3)$ or before use of PT ( $n=3$ ); use of ET before use of PT ( $n=3$ ); use of PT before use of ET (n $=2)$, and unknown start dates for either ET or PT (n=33) for a total of 134 women. The baseline questionnaire assessed demographics, body mass index (BMI), reproductive history, oral contraceptive use, menopausal status, family history of cancer, and smoking status, which were all considered potential confounders.

\section{Statistical analysis}

Multivariable Cox proportional hazards regression models were used to estimate adjusted hazard ratios (HRs) and 95\% confidence intervals (CIs) for five- and 10-year all-cause and endometrial cancer-specific mortality with age as the underlying time metric. Follow-up time started at the age endometrial cancer was diagnosed and ended at the age that death occurred or age at end of follow-up (December 31, 2011), whichever occurred first. The proportional hazards assumption was evaluated with likelihood ratio tests comparing models with and without interaction terms between MHT use and follow-up time.

We examined relationships between MHT formulation (no MHT, ET, EPT-only, combinations of ET and EPT), regimen of EPT (sequential or continuous), and recency of use (ET and EPT-only formulations in separate models) with all-cause and endometrial cancer-specific mortality. Recency of MHT use refers to status at the time of the second risk factor questionnaire, not the endometrial cancer diagnosis. Indicator variables for other MHT formulations were included in each model. Tests for linear trend were conducted by entering the categorical variables of recency into the model as ordinal variables.

We first built a base model including MHT formulation, stage (localized, regional, distant), joint histology and tumor grade (low-grade endometrioid, high-grade endometrioid, serous, other), surgery (yes, no), radiotherapy (yes, no), and chemotherapy (yes, no). Variables listed in Table 1 were included in the final model if their addition changed the base model estimates by more than $10 \%$ or if the model fit was significantly improved as assessed by the likelihood ratio test. Thus, the final model included additional adjustment for race, (white, non-white), BMI at baseline ( $\left.<25,25-29.9,30-34.9,35-39.9, \geq 40 \mathrm{~kg} / \mathrm{m}^{2}\right)$, smoking status (never, former, current), and diabetes (yes, no). Missing data were treated as separate 
categories for relevant variables. Effect modification was examined by creating multiplicative interaction terms between MHT formulation and a priori effect modifiers [age at MHT assessment, BMI (3-level categorical variable), stage, and joint histology and tumor grade] and calculating likelihood ratio test statistics comparing models with and without the interaction terms.

We performed several sensitivity analyses. Time between completion of the second risk factor questionnaire and endometrial cancer diagnosis (lag time) varied; therefore, we categorized women into three time blocks by year of diagnosis (1995-1998, 1999-2002, 2003-2006) and tested for interaction with MHT formulation. To account for potential changes in MHT prescribing patterns that may have occurred after publication of the Women's Health Initiative (WHI) results, we truncated follow-up time for all women on June 30, 2002, a date just prior to the release of the WHI results. Finally, we ran analyses excluding women diagnosed with endometrial cancer after December 31, 2001, as these women did not have an opportunity to contribute 10 years of follow-up to the long-term mortality analysis. All analyses were performed using SAS 9.3 software (SAS Institute Inc., Cary, NC).

\section{RESULTS}

\section{Cohort characteristics}

Our cohort consisted of 890 epithelial endometrial cancer cases diagnosed a median of 4.6 years (range $0.0-10.1$ years) after the second risk factor questionnaire was completed. Fifty-six percent $(\mathrm{n}=498)$ of endometrial cancer cases reported no pre-diagnosis MHT use, whereas $8.1 \%(\mathrm{n}=72)$ reported using ET, 20.9\% ( $\mathrm{n}=186)$ reported using EPT-only, and $15.1 \%(\mathrm{n}=134)$ reported combinations of ET and EPT. Baseline characteristics of the cohort according to MHT formulation are shown in Table 1. Compared with non-MHT users, endometrial cancer cases who used ET prior to their diagnosis tended to be older at enrollment, have post-high school or some college education, have normal BMI, have used oral contraceptives, and were less likely to have regional or distant stage cancers. Prediagnosis users of EPT-only had similar characteristics as their ET counterparts except they were younger at enrollment and less likely to have a history of diabetes compared with nonMHT users.

\section{Mortality during follow-up by MHT formulation}

For brevity, we present results for 10-year mortality as we did not observe relationships with five-year mortality. Median follow-up time from the endometrial cancer diagnosis to death or end of follow-up was 8.8 years (range: 0 - 15.1 years). Through 2011, a total of 241 deaths were identified, of which 104 were due to endometrial cancer. Table 2 presents the association between pre-diagnosis MHT formulation and 10-year all-cause and endometrial cancer-specific mortality. More than $40 \%$ of the endometrial cancer cases in this cohort had follow-up of 10 or more years. No association between ET and mortality was observed. Compared with non-MHT users, cases who reported using EPT-only had lower all-cause $(\mathrm{HR}=0.65,95 \% \mathrm{CI}=0.43-0.99)$ and endometrial cancer-specific mortality $(\mathrm{HR}=0.51,95 \%$ $\mathrm{CI}=0.26-0.98)$. An examination of specific EPT regimens did not reveal significant 
associations related to sequential or continuous use of EPT; however, these analyses were based on small numbers of deaths.

Table 3 presents HRs for associations between recency of ET and EPT-only with mortality. Recency of use refers to MHT status based on responses from the second risk factor questionnaire. Compared with non-MHT users, former ET users had higher all-cause ( $\mathrm{HR}=1.71,95 \% \mathrm{CI}=1.02-2.88$, based on 18 deaths) and endometrial cancer-specific mortality ( $\mathrm{HR}=2.17,95 \% \mathrm{CI}=0.96-4.90$, based on 8 deaths). No significant trend in recency of ET was observed for 10-year all-cause ( $\mathrm{p}$-trend=0.53) or endometrial cancer-specific mortality (p-trend=0.46). In analyses of EPT-only recency, sequential and continuous EPT users were combined. Women who reported current EPT use on the second risk factor questionnaire had lower 10-year all-cause $(\mathrm{HR}=0.67,95 \% \mathrm{CI}=0.43-1.05$, $\mathrm{p}$-trend $=0.05)$ and endometrial cancer-specific mortality $(\mathrm{HR}=0.54,95 \% \mathrm{CI}=0.27-1.11$, $\mathrm{p}$-trend $=0.06$ ); however, these results are based on 26 and 10 deaths respectively.

\section{Additional analyses}

We did not observe that the relationship between MHT formulation and 10-year mortality varied significantly according to the presence or absence of a number of risk factors and clinical characteristics of the tumors (Table 4). However, we did note that EPT was associated with improved survival in young women and obese women. Furthermore, we did not observe significant heterogeneity of MHT formulations by lag time for 10-year all-cause $\left(p_{\text {interaction }}=0.22\right)$ or 10 -year endometrial cancer-specific mortality $\left(p_{\text {interaction }}=0.36\right)$ (Supplementary Table 1). In analyses where follow-up was ended on June 30, 2002 to account for potential changes in MHT use after the release of the WHI results, patterns of association were similar to the main results, but non-significant, due to the lower number of cases included in the model (data not shown). Further, as women diagnosed after December 31, 2001 did not have an opportunity to contribute a full 10 years of follow-up to the longterm mortality analysis, we examined models restricted to women diagnosed with endometrial cancer between the second risk factor questionnaire and December 31, 2001. Despite small numbers, we observed similar patterns of association in these analyses as compared with analyses including all endometrial cancer cases (data not shown).

\section{DISCUSSION}

In this prospective study of women diagnosed with endometrial cancer, pre-diagnosis use of EPT was inversely associated with 10-year all-cause and endometrial cancer-specific mortality. When examined by recency of use, endometrial cancer cases with more proximate exposure to EPT had borderline lower risks of dying; however these results were based on small numbers of deaths. Additionally, endometrial cancer cases reporting former ET exposure on the second risk factor questionnaire had higher risks of death compared with endometrial cancer cases that did not use MHT.

Only one previous study has examined EPT and outcomes among endometrial cancer cases [13]. Relative to survival in the general Swedish population, neither EPT (all durations of progestin combined) nor sequential EPT were associated with survival in endometrial cancer cases. Similar to our study, the prior analysis was limited by a small numbers of EPT users 
which may have reduced their ability to detect associations between EPT users and nonusers. Our study provides evidence that EPT, which is associated with lower risk of developing endometrial cancer [3], is also inversely associated with mortality following an endometrial cancer diagnosis.

To date, the literature has been inconsistent regarding the relationship between pre-diagnosis ET use and mortality among women with endometrial cancer [5-13]. Some studies have shown that pre-diagnosis ET use is not associated with mortality after accounting for tumor grade $[5,6]$ while others report lower mortality associated with ET use after adjustment for grade $[9,11]$. Overall, we did not observe an association between pre-diagnosis ET use and mortality among endometrial cancer cases in models that were unadjusted for tumor characteristics (stage or the joint histology and tumor grade variable) or in fully adjusted models. Based on small numbers, we did observe higher risks of death associated with former use of ET. These results should be interpreted cautiously, as we lacked information on the exact timing of MHT use in relation to the endometrial cancer diagnosis. Our method of ascertainment differs from those studies that assessed ET use through personal interviews soon after the endometrial cancer diagnosis $[9,11]$ or through medical record review [6]. Further, changes in the type and dose of ET have occurred over time [20] which may be a contributing factor to differences between our results and the previous literature, as our study included endometrial cancer patients diagnosed between 1996 and 2006 while the previous literature included endometrial cancer patients diagnosed in the 1970s and 1980s.

MHT use may affect endometrial cancer mortality through a number of mechanisms. One such mechanism is through promotion of favorable tumor characteristics. Stage, grade and tumor subtype are strongly related to mortality among endometrial cancer cases [21]. Some have noted that MHT use, specifically ET, is associated with early stage and well differentiated tumors $[6,9,13,22-25]$. Conversely, the WHI randomized trial did not report differences in these characteristics between users and non-users of MHT [26]. In our study, the distribution of stage significantly differed across MHT formulations. EPT users and nonMHT users had a similar prevalence of localized tumors, whereas ET users had a lower prevalence of localized tumors, which contradicts previous studies. We observed no difference in the combined histology/tumor grade variable by MHT formulation. Importantly, the addition of these variables to the model did not change the association between MHT formulation and mortality. If MHT affected mortality only by producing favorable tumor characteristics, we would expect this survival advantage to be eliminated after statistical adjustment for these characteristics. Further, we did not observe major differences in the association between MHT formulation and mortality when we stratified by these tumor characteristics.

Increased medical surveillance has been offered as an explanation for better survival outcomes among MHT users [5, 9]. MHT users may have a higher frequency of encounters with medical providers compared with non-MHT users, which may result in the detection of early stage tumors, and hence, more favorable prognosis. As mentioned above, we observed similar proportions of localized tumors among EPT users and non-MHT users. Therefore, our findings do not support the theory that increased surveillance, with earlier detection of endometrial tumors, is responsible for the association between EPT use and lower mortality. 
Some have noted that MHT users are more educated and affluent than women in the general population [20, 27], which may influence outcomes. Further, MHT users tend to participate in more health promoting activities such as maintaining a healthy body weight and engaging in physical activity [20]. We observed these characteristics in our study population; women who used MHT were more likely to have more years of education and normal BMI, but less likely to have diabetes. Importantly, only some of these variables proved to be important confounders in our study population, and adjustment for these characteristics did not alter our associations. There may, however, be residual confounding by income, access to health care, and other factors not directly measured in this study. Additionally, there is a possibility that selection factors regarding the prescription of MHT influenced our results. For example, hypertension and heart disease are associated with higher mortality, yet these diseases are contraindications to MHT prescription. Consequently, if women with these conditions were excluded from our study population of MHT users, the risk of dying among MHT users would be downwardly biased to be more protective.

The major strength of our study is the use of a prospective cohort to assess associations between MHT formulation and mortality outcomes among endometrial cancer cases, which has been inadequately addressed in the literature. Furthermore, our outcomes were standardly assessed and we were able to control for other factors related to mortality among endometrial cancer patients. The major limitation of our study is our assumption that MHT status, which was self-reported on the 1996 risk factor questionnaire, remained constant during the follow-up period. Other studies have shown the WHI results produced a dramatic decline in MHT use [28]. Our analyses limited to events occurring before this date show no association between EPT-only MHT and mortality, potentially reflecting limited statistical power. Although a third risk factor questionnaire assessing MHT use was sent to participants in 2004, the frequency of responses related to MHT use was too sparse to update MHT status. Other limitations include the small numbers of deaths in certain exposure categories, use of self-reported risk factor information that was not updated over follow-up (e.g. BMI), limited follow-up among women diagnosed after December 31, 2001, and lack of detailed information on treatment. As treatment recommendations for early stage endometrial cancer are uniform, we do not expect treatment differences to play a major role in our findings.

In summary, pre-diagnosis use of EPT was associated with lower 10-year all-cause and endometrial cancer-specific mortality. Future studies examining the biological mechanisms underlying this association are warranted. Our results may be of use to clinicians that treat endometrial cancer patients with previous exposure to MHT.

\section{Supplementary Material}

Refer to Web version on PubMed Central for supplementary material.

\section{Acknowledgments}

Financial support: This research was supported (in part) by the Intramural Research Program of the National Cancer Institute. 


\section{References}

1. Brinton LA, Felix AS. Menopausal hormone therapy and risk of endometrial cancer. J Steroid Biochem Mol Biol. 2013

2. Grady D, Gebretsadik T, Kerlikowske K, Ernster V, Petitti D. Hormone replacement therapy and endometrial cancer risk: a meta-analysis. Obstet Gynecol. 1995; 85(2):304-13. [PubMed: 7824251]

3. Trabert B, Wentzensen N, Yang HP, Sherman ME, Hollenbeck AR, Park Y, Brinton LA. Is estrogen plus progestin menopausal hormone therapy safe with respect to endometrial cancer risk? Int J Cancer. 2013; 132(2):417-26. [PubMed: 22553145]

4. Beral V, Bull D, Reeves G. Endometrial cancer and hormone-replacement therapy in the Million Women Study. Lancet. 2005; 365(9470):1543-51. [PubMed: 15866308]

5. Robboy SJ, Bradley R. Changing trends and prognostic features in endometrial cancer associated with exogenous estrogen therapy. Obstet Gynecol. 1979; 54(3):269-77. [PubMed: 471366]

6. Smith DC, Prentice RL, Bauermeister DE. Endometrial carcinoma: histopathology, survival, and exogenous estrogens. Gynecol Obstet Invest. 1981; 12(4):169-79. [PubMed: 7250780]

7. Petitti DB, Perlman JA, Sidney S. Noncontraceptive estrogens and mortality: long-term follow-up of women in the Walnut Creek Study. Obstet Gynecol. 1987; 70(3 Pt 1):289-93. [PubMed: 3627576]

8. Underwood PB Jr, Miller MC, Kreutner A Jr, Joyner CA, Lutz MH. Endometrial carcinoma: the effect of estrogens. Gynecol Oncol. 1979; 8(1):60-73. [PubMed: 456937]

9. Collins J, Donner A, Allen LH, Adams O. Oestrogen use and survival in endometrial cancer. Lancet. 1980; 2(8201):961-4. [PubMed: 6107599]

10. Chu J, Schweid AI, Weiss NS. Survival among women with endometrial cancer: a comparison of estrogen users and nonusers. Am J Obstet Gynecol. 1982; 143(5):569-73. [PubMed: 7091228]

11. Schwartzbaum JA, Hulka BS, Fowler WC Jr, Kaufman DG, Hoberman D. The influence of exogenous estrogen use on survival after diagnosis of endometrial cancer. Am J Epidemiol. 1987; 126(5):851-60. [PubMed: 3661533]

12. Paganini-Hill A, Ross RK, Henderson BE. Endometrial cancer and patterns of use of oestrogen replacement therapy: a cohort study. Br J Cancer. 1989; 59(3):445-7. [PubMed: 2930713]

13. Orgeas CC, Hall P, Wedren S, Dickman PW, Czene K. The influence of menopausal hormone therapy on tumour characteristics and survival in endometrial cancer patients. Eur J Cancer. 2009; 45(17):3064-73. [PubMed: 19493676]

14. Schatzkin A, Subar AF, Thompson FE, Harlan LC, Tangrea J, Hollenbeck AR, et al. Design and serendipity in establishing a large cohort with wide dietary intake distributions : the National Institutes of Health-American Association of Retired Persons Diet and Health Study. Am J Epidemiol. 2001; 154(12):1119-25. [PubMed: 11744517]

15. Michaud D, Midthuner D, Hermansen S, Leitzmann MF, Harlan LC, Kipnis V, Schatzkin A. Comparison of Cancer Registry Case Ascertainment with SEER Estimates and Self-reporting in a subset of the NIH-AARP Diet and Health Study. J Registry Manag. 2005; 32(2):6.

16. Zaino RJ. FIGO staging of endometrial adenocarcinoma: a critical review and proposal. Int J Gynecol Pathol. 2009; 28(1):1-9. [PubMed: 19047915]

17. Hermansen SW, Leitzmann MF, Schatzkin A. The impact on National Death Index ascertainment of limiting submissions to Social Security Administration Death Master File matches in epidemiologic studies of mortality. Am J Epidemiol. 2009; 169(7):901-8. [PubMed: 19251755]

18. Lacey JV Jr, Brinton LA, Lubin JH, Sherman ME, Schatzkin A, Schairer C. Endometrial carcinoma risks among menopausal estrogen plus progestin and unopposed estrogen users in a cohort of postmenopausal women. Cancer Epidemiol Biomarkers Prev. 2005; 14(7):1724-31. [PubMed: 16030108]

19. Sorensen HT, Sabroe S, Olsen J. A framework for evaluation of secondary data sources for epidemiological research. Int J Epidemiol. 1996; 25(2):435-42. [PubMed: 9119571]

20. Nelson HD, Humphrey LL, Nygren P, Teutsch SM, Allan JD. Postmenopausal hormone replacement therapy: scientific review. JAMA. 2002; 288(7):872-81. [PubMed: 12186605] 
21. Creasman WT, Odicino F, Maisonneuve P, Quinn MA, Beller U, Benedet JL, et al. Carcinoma of the corpus uteri. FIGO 26th Annual Report on the Results of Treatment in Gynecological Cancer. Int J Gynaecol Obstet. 2006; 95(Suppl 1):S105-43. [PubMed: 17161155]

22. Mittal KR, Barwick KW. Endometrial adenocarcinoma involving adenomyosis without true myometrial invasion is characterized by frequent preceding estrogen therapy, low histologic grades, and excellent prognosis. Gynecol Oncol. 1993; 49(2):197-201. [PubMed: 8504988]

23. Nyholm HC, Nielsen AL, Norup P. Endometrial cancer in postmenopausal women with and without previous estrogen replacement treatment: comparison of clinical and histopathological characteristics. Gynecol Oncol. 1993; 49(2):229-35. [PubMed: 8504992]

24. Shapiro JA, Weiss NS, Beresford SA, Voigt LF. Menopausal hormone use and endometrial cancer, by tumor grade and invasion. Epidemiology. 1998; 9(1):99-101. [PubMed: 9430277]

25. Chapman JA, DiSaia PJ, Osann K, Roth PD, Gillotte DL, Berman ML. Estrogen replacement in surgical stage I and II endometrial cancer survivors. Am J Obstet Gynecol. 1996; 175(5):1195200. [PubMed: 8942487]

26. Anderson GL, Judd HL, Kaunitz AM, Barad DH, Beresford SA, Pettinger M, et al. Effects of estrogen plus progestin on gynecologic cancers and associated diagnostic procedures: the Women's Health Initiative randomized trial. JAMA. 2003; 290(13):1739-48. [PubMed: 14519708]

27. Matthews KA, Kuller LH, Wing RR, Meilahn EN, Plantinga P. Prior to use of estrogen replacement therapy, are users healthier than nonusers? Am J Epidemiol. 1996; 143(10):971-8. [PubMed: 8629615]

28. Hersh AL, Stefanick ML, Stafford RS. National use of postmenopausal hormone therapy: annual trends and response to recent evidence. JAMA. 2004; 291(1):47-53. [PubMed: 14709575] 


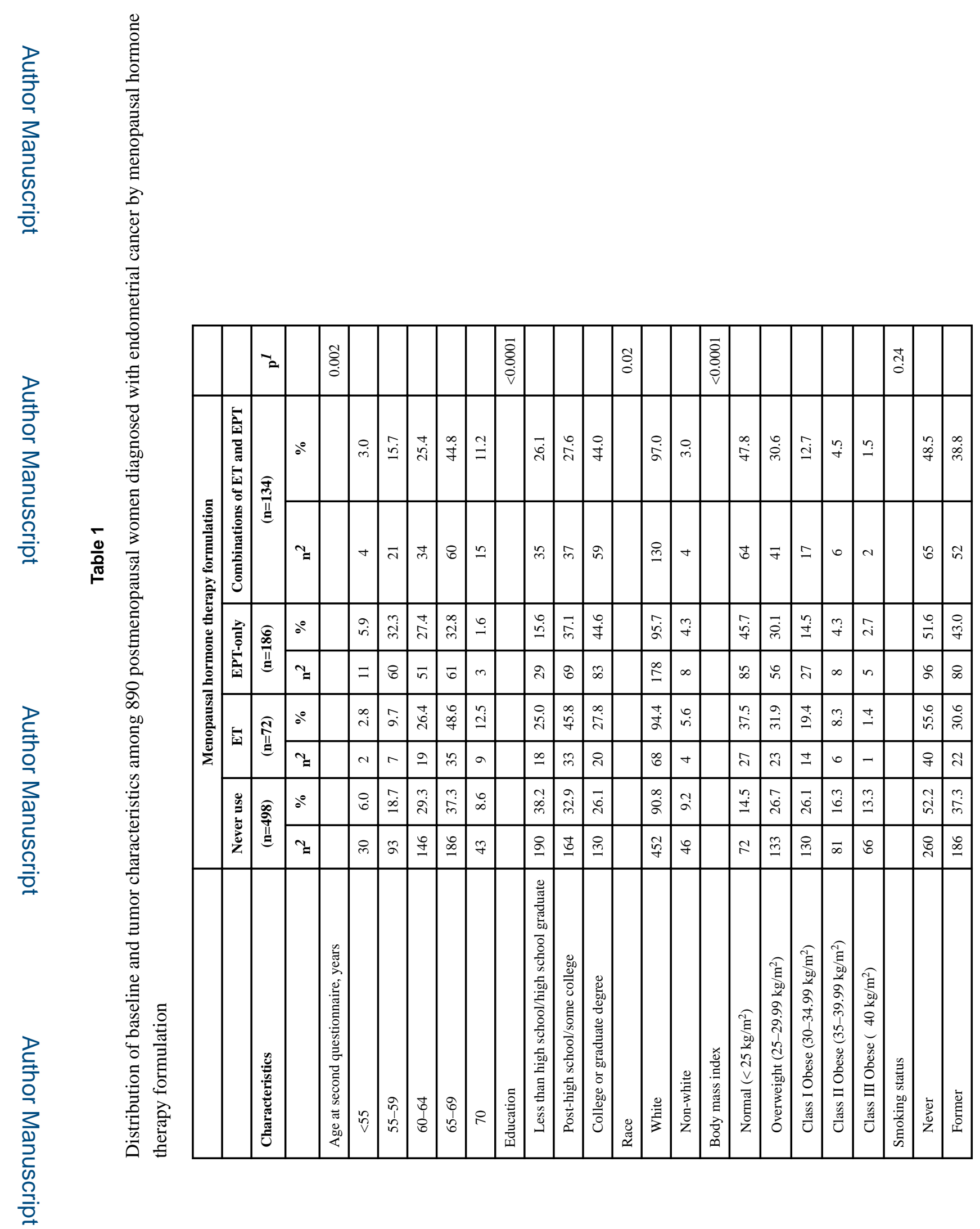

Cancer Causes Control. Author manuscript; available in PMC 2016 December 16. 


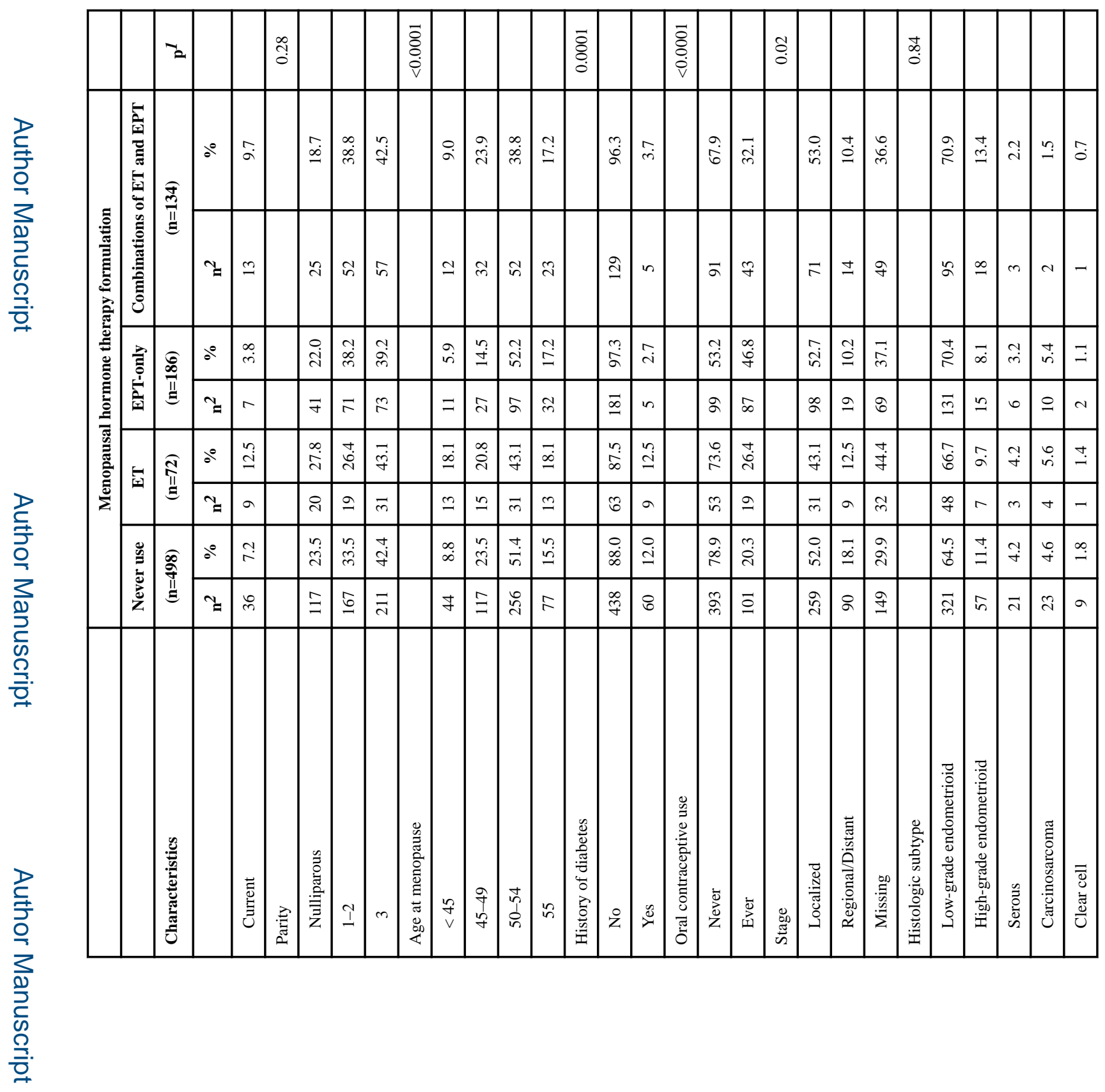

로을 


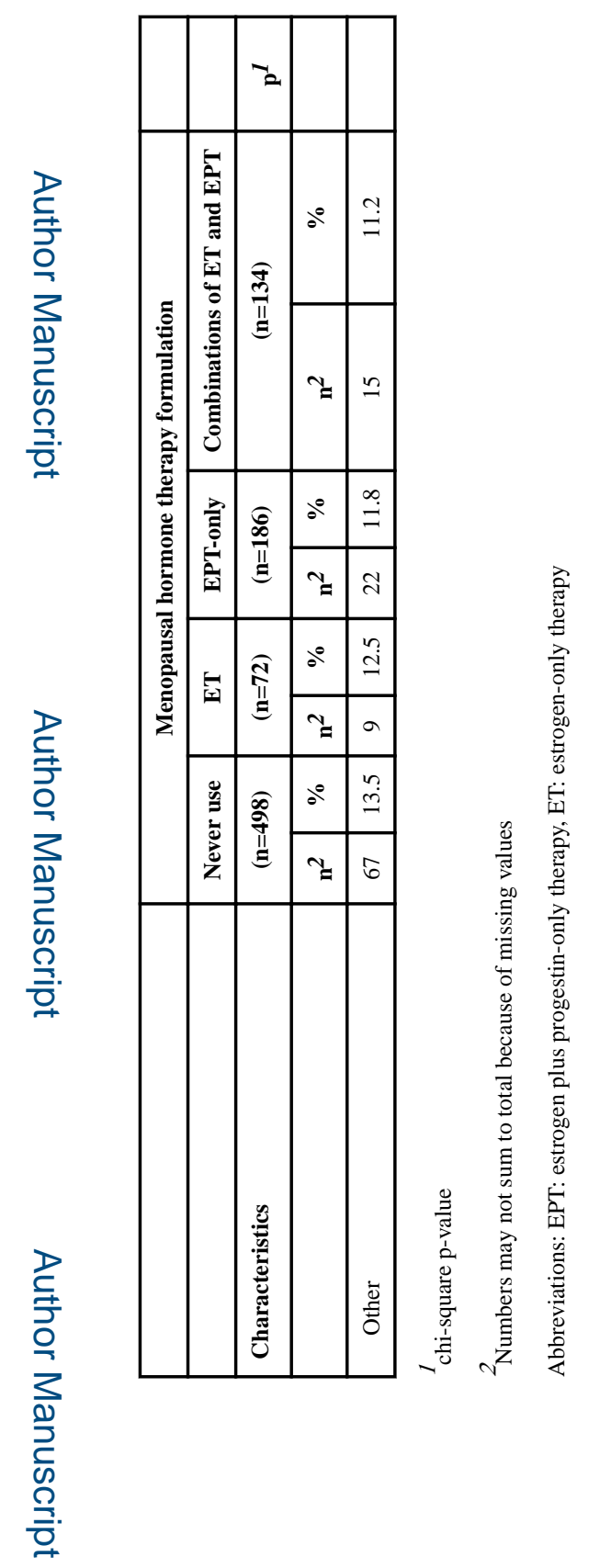

롤 


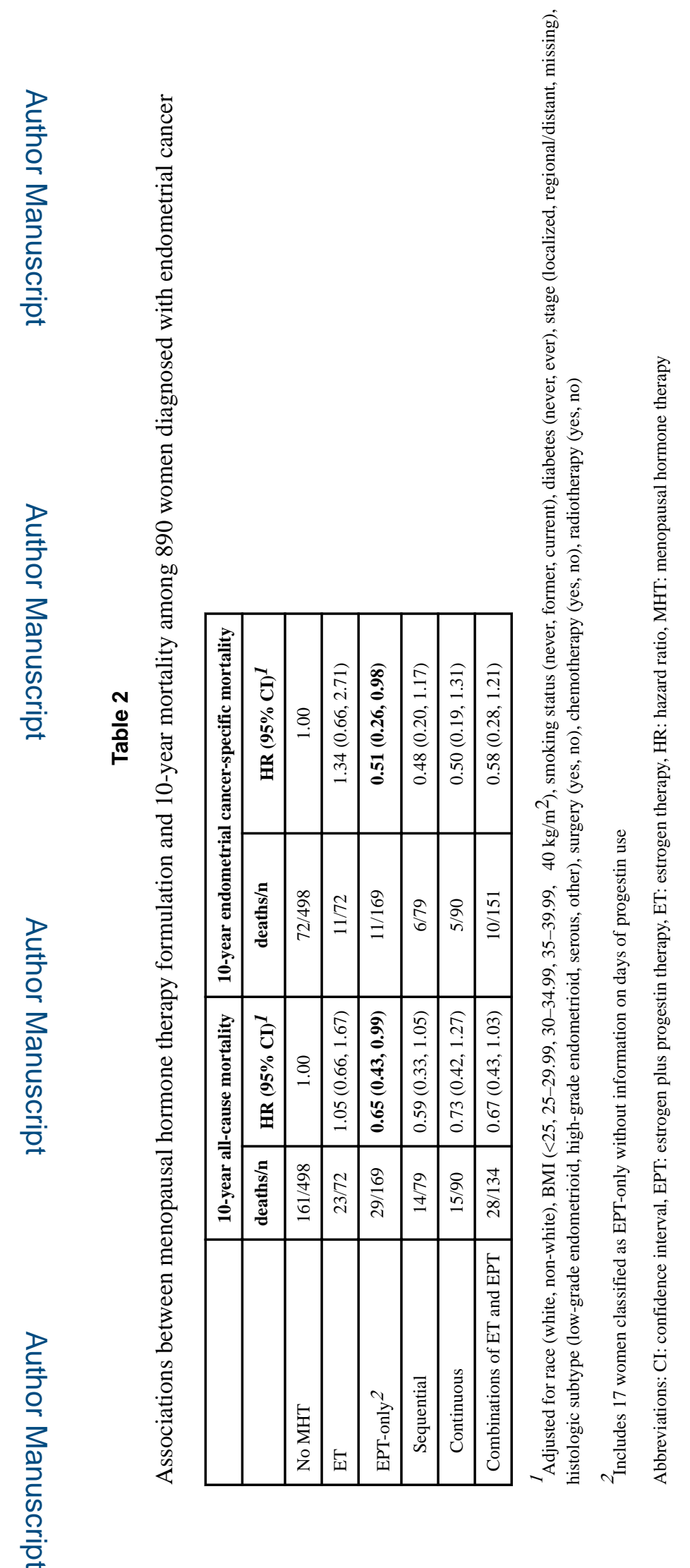

Cancer Causes Control. Author manuscript; available in PMC 2016 December 16. 


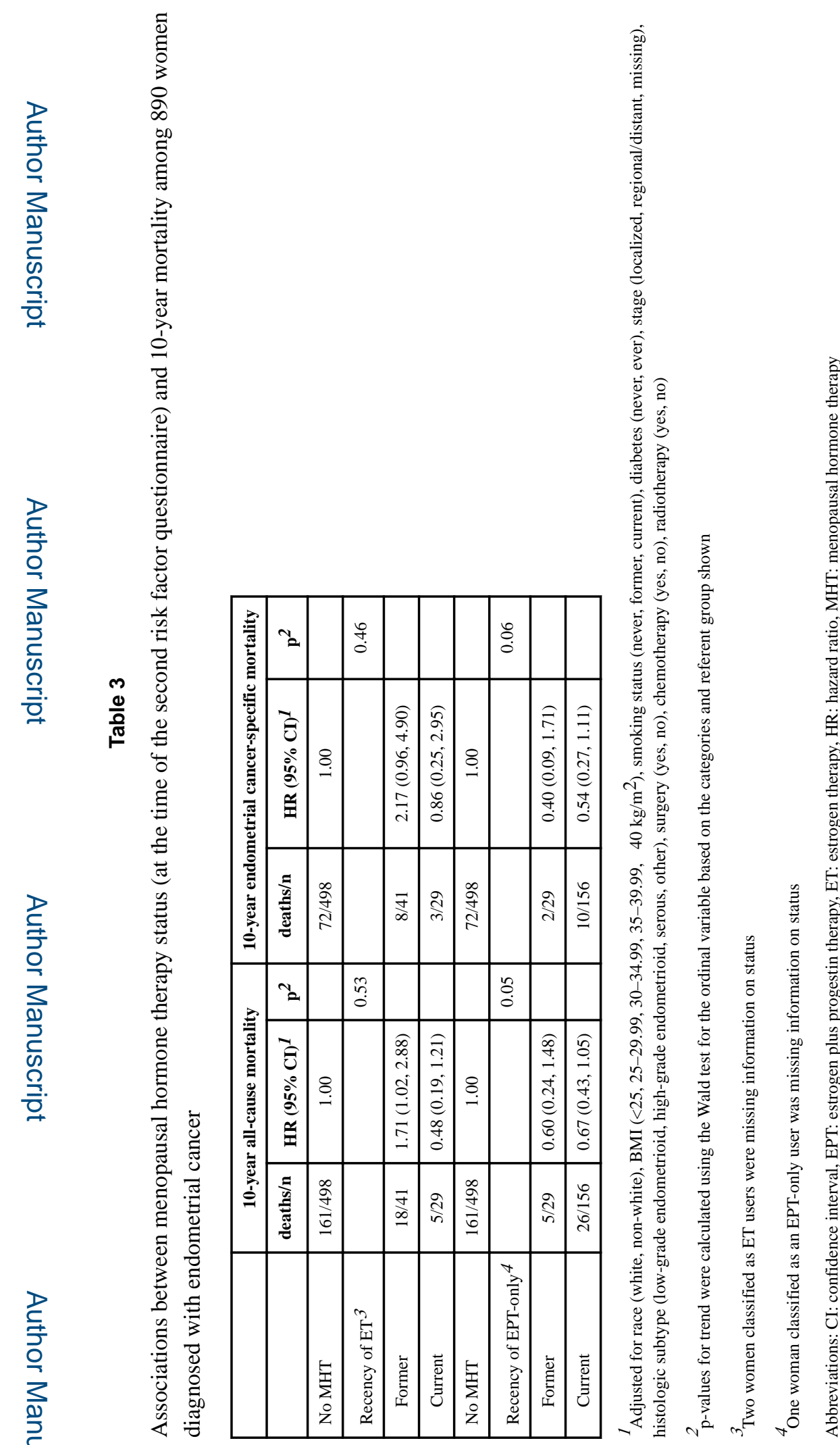




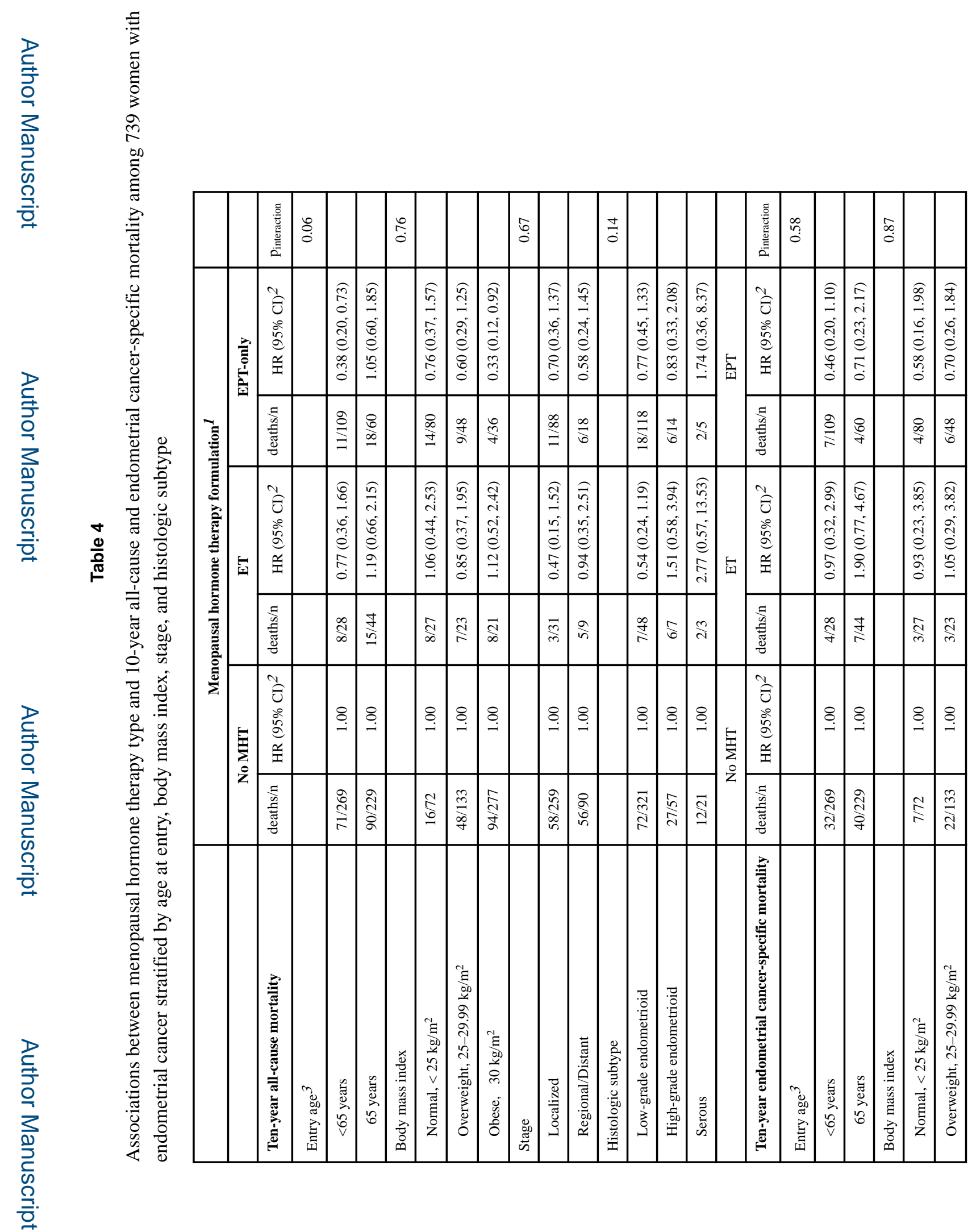

Cancer Causes Control. Author manuscript; available in PMC 2016 December 16. 


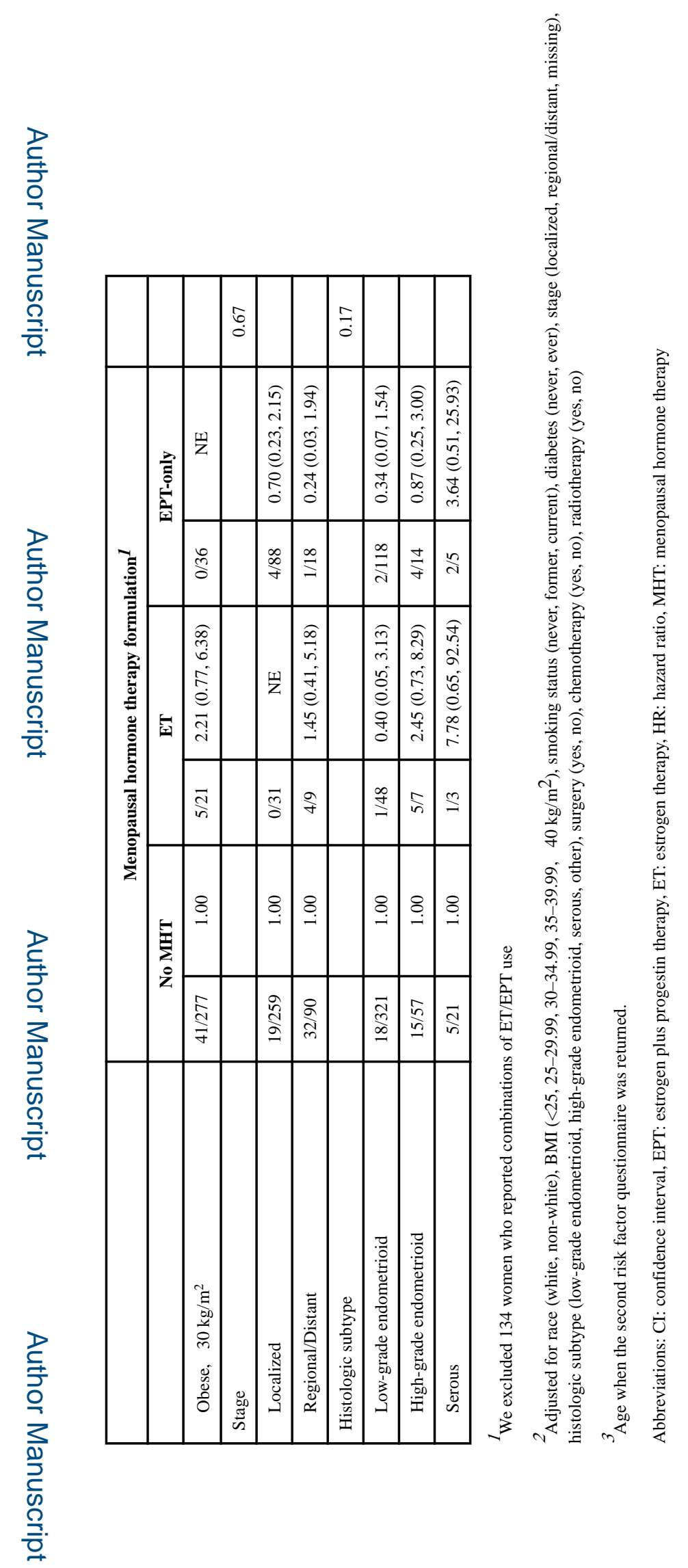

Cancer Causes Control. Author manuscript; available in PMC 2016 December 16. 